
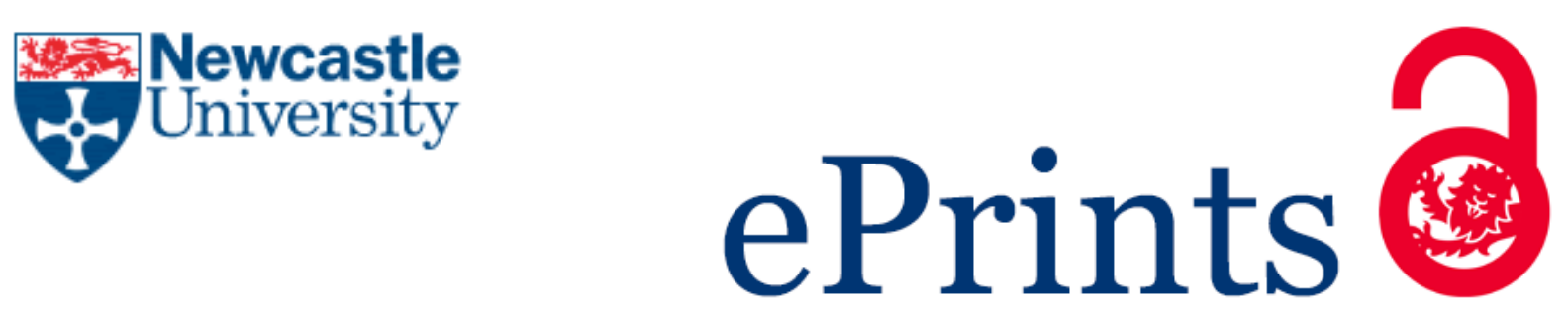

Hartai M, Nyari TA, McNally RJ.

Trends in mortality rates in female genital organs and breast cancers in

Hungary between 1979 and 2013.

European Journal of Obstetrics \& Gynecology and Reproductive

Biology 2015, 194, 168-172.

Copyright:

(C) 2015. This manuscript version is made available under the CC-BY-NC-ND 4.0 license

DOI link to article:

http://dx.doi.org/10.1016/i.ejogrb.2015.08.021

Date deposited:

$21 / 12 / 2015$

Embargo release date:

18 September 2016

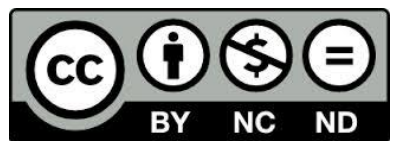

This work is licensed under a

Creative Commons Attribution-NonCommercial-NoDerivatives 4.0 International licence 
Trends in mortality rates in female genital organs and breast cancers in Hungary between 1979 and 2013

\author{
Margit Hartai ${ }^{1}$, Tibor András Nyári ${ }^{1 * a}$, Richard J.Q, McNally ${ }^{2 a}$
}

${ }^{1}$ Department of Medical Physics and Informatics, University of Szeged, Hungary

${ }^{2}$ Institute of Health and Society, Newcastle University, United Kingdom

${ }^{a}$ Authors contributed equally to this work.

Short title: Trends in female cancers mortality

Paper type: regular article

Number of WORDs : 2207

Abstract: 215 words

*Correspondence author: Tibor András Nyári; University of Szeged; Department of Medical Physics and Informatics; H-6701, Szeged, P.O. Box: 427; Hungary

Tel./Fax: +36-62-544566

E-mail: Nyari.Tibor@med.u-szeged.hu 


\section{Condensation}

This epidemiological study reports significant seasonal trends with peaks in August in mortality in female genital organs and breast cancer. 


\title{
Trends in mortality rates in female genital organs and breast cancers in Hungary between 1979 and 2013
}

\author{
Margit Hartai, Tibor András Nyári, Richard J.Q, McNally
}

\begin{abstract}
Objective: This study investigated annual and seasonal trends in deaths for cancers of the female genital organs and breast in Hungary between 1979 and 2013.

Study Design: Data on the numbers of cancer deaths were obtained from the published nationwide population register. Joinpoint regression was applied to investigate the yearly trends in cancer mortality rates. Cyclic trends were investigated using logistic regression, Edwards' and Walter-Elwood methods.
\end{abstract}

Results: The majority of deaths from cancers of the female genital organs and breast occurred in winter but using the observed numbers of deaths a significant seasonal pattern was only revealed for deaths from breast cancer with a peak in January and a nadir in July. However, seasonality in the proportion of deaths from female genital organs and breast cancers out of deaths from all causes detected a different peak and nadir. The proportion of female genital organs and breast cancer deaths out of deaths from all causes was higher around the end of summer and significant seasonal variation with a peak in August and nadir in February was revealed.

Conclusion: This Hungarian study suggests that there was a significant seasonal effect on female genital organs and breast cancer mortality. Both seasonal patterns are interesting and informative to potentiate prevention. Our findings suggest that infectious diseases may increase the risk of the mortality among the immune deficient patents.

Keywords: Hungary, breast cancer mortality, gynecological cancer mortality, seasonal variations 


\section{Abbreviations}

ASMRs age-standardized mortality rates

ICD10 International Statistical Classification of Diseases and Related Health Problems

10th Revision

RR relative risk

$95 \%$ CI $\quad 95 \%$ confidence interval 


\section{Introduction}

Breast and cervical cancers are both common malignancies in Hungarian women. In Hungary, annually approximately 5500 and 1200 new cases are diagnosed with breast and cervical cancer, respectively [1]. Furthermore, the mortality rate of breast cancer is high in Hungary as the vast proportion of these cases are diagnosed too late [2-3]. Similarly, mortality rates for corpus uteri and ovarian cancers are in a higher rank than in the other countries of the European Union [4]. The susceptibility to an infectious disease is higher among cancer patients as their immune system is suppressed. If seasonal variation in the onset of disease could be shown in any of the diagnostic subgroups, this could be interpreted as supportive evidence for an etiology linked to exposure to infection [5].

In this study, together with analyses of changes in mortality rates for malignant neoplasms of female genital organs and breast, the annual and seasonal trends in mortality rates are investigated in Hungary between 1979 and 2013.

\section{Material and methods}

\section{Study population}

The 35-year period between 1979 and 2013 was considered in this analysis. Data on the population and numbers of deaths from malignant neoplasms of female genital organs and breast were obtained from the published nationwide population register of the Hungarian Central Statistical Office [6].

The data concerning cause of deaths were published annually by the Central Statistical Office. These data were classified according to the ICD10 codes. The main categories were: malignant neoplasms of breast (ICD10: C50), malignant neoplasm of cervix uteri (ICD10:C53), malignant neoplasm of uterus: corpus uteri (ICD10:C54) part unspecified (ICD10:C55) and malignant 
neoplasm of ovary (ICD10:C56). Data on both the population and numbers of deaths from cancer were classified by age groups as follow: 0-14 years, 15-39 years, 40-59 years, 60-69 years, 70-79 years and over 80 years. Thus, age-specific death rates were calculated. Furthermore, the mortality rates of cancer of the female genital organs and breast were directly standardized using the standard world population to make comparisons easier. Since the numbers of deaths were too small in the group aged 0-14 years, all analyses were carried out omitting this group. There were no data on malignant neoplasm of vulva (ICD10:C51), vagina (ICD10:C52), placenta (ICD10:C58) and other and unspecified female genital organs (ICD10:C57).

\section{Investigation of seasonal trends}

The numbers of monthly deaths from cancers of the female genital organs and breast have been available since 1979 [6]. Also data on the total numbers of monthly deaths from all causes were available for each year of the study period and were obtained from the published nationwide population register. Although the ICD coding underwent change, data on deaths from cancer of the female genital organs and breast were consistently available during the study period.

\section{Statistical methods}

\section{Investigation of annual trend}

Age-standardized mortality rates (ASMRs) were used to investigate trends in mortality rates for malignant neoplasms of the female genital organs and breast in Hungary.

Trends were investigated using negative binomial regression models in analyses by age group for cancers of the female genital organs and breast. Relative risks (RR) and 95\% confidence intervals (95\% CIs) were calculated. Joinpoint regression analyses were additionally applied to characterize trends and to find turning points during the period 1979-2013. Joinpoint analyses 
were done using the joinpoint regression software (version 4.0.4) obtained from the Surveillance Research Program of the US National Cancer Institute [7]

\section{Investigation of seasonal trends}

Data on the month of the death were aggregated over the study period. Cyclical trends in these monthly data were investigated using the Edwards' test [8], the Walter-Elwood method [9] and the logistic regression method [5] using both sine and cosine functions. Monthly cyclical trends (seasonality) were investigated in the following groups: deaths from malignant neoplasms of the breast, cervix uteri, uterus and ovary, respectively.

$P$-values less than 0.05 were considered to be statistically significant. All analyses were performed using STATA Software version 12.0 (Stata Corp LP, College Station, Texas, USA)

\section{Results}

Overall, there were 74 920, 18 610, 22885 and 19472 deaths from breast cancer, cervix cancer, corpus uteri cancer and ovarian cancer, respectively, in Hungary during the period 1979-2013. Most deaths occurred from breast cancer (Table 1). The crude mortality rates were 39.661 per 100000 persons per year, 9.916 per 100000 persons per year, 12.193 per 100000 persons per year and 10.291 per 100000 persons per year for breast cancer, cervix cancer, corpus uteri cancer and ovarian cancer. After direct standardization these rates became 24.254 per 100000 persons per year, 6.893 per 100000 persons per year, 7.449 per 100000 persons per year and 5.721 per 100000 persons per year for breast cancer, cervix cancer, corpus uteri cancer and ovarian cancer. 
The smallest age-standardized mortality rates for malignant neoplasms of the female genital organs and breast were observed in Hungary in 2009. Annual age-standardized mortality rates between 19.557 per 100000 persons per year (2009) and 27.751 per 100000 persons per year (1994) were observed in breast cancer mortality (Figure 1). For malignant neoplasms of the female genital organs the annual ASMRs varied between 4.553 per 100000 persons per year (2009) and 9.332 per 100000 persons per year (1982) in cervix cancer mortality, between 9.571 per 100000 persons per year (1979) and 2.961 per 100000 persons per year (2009) in corpus uteri cancer mortality and between 8.905 per 100000 persons per year (1980), and 6.113 per 100000 persons per year (1979) in ovarian cancer mortality(Figure 1).

Although the highest age-specific mortality rates were observed in the group aged over 80 years, however, after standardization the highest ASMRs were observed in the group aged 4059 years (Table 2). The ASMRs decreased in the older age groups for all malignant neoplasms of the female genital organs and breast.

\section{Annual trends in mortality}

The negative binomial regression model for annual age-standardized data revealed a declining trend in the 1 yearly ASMRs for cervical cancer (RR 0.981 95\%CI [0.968-0.993]; $\mathrm{p}=0.003$ ) and corpus uteri cancer $(\mathrm{RR}: 0.968,95 \% \mathrm{CI}[0.954-0.981] \mathrm{p}<0.001)$ during the study period but no linear trend was found for breast cancer and ovarian cancer mortality.

Similarly, decreasing annual trends in the ASMRs of cervical cancer and corpus uteri cancer (Figure 1b) were found using joinpoint regression during the study period. However, a slightly increasing trend in the ASMRs of corpus uteri cancer was detected (Figure 1c).

The joinpoint analyses depicted turning points in 1998 and 2009 for breast cancer mortality and changes in segmented linear trends (Figure 1a-1d). The annual changes in percentages and 
slopes of the segments in the deaths from breast cancer for 1979-1998 were: $0.89 \%(\mathrm{p}=0.001)$, for 1998-2009: $-2.8 \%(\mathrm{p}=0.001)$ and for $2009-2013: 1.1 \%(\mathrm{p}=0.08)$.

The annual changes in percentages and slopes of the segments in the deaths from ovarian cancer for 1979-2009 were: $0.9 \%(\mathrm{p}<0.001)$ and for 2009-2013: $3.8 \%(\mathrm{p}=0.072)$.

\section{Seasonal trends}

The aggregated numbers of deaths are summarized in Table 3. The overall proportion of the deaths from breast cancer was the highest among all deaths (Table 3). Using the Edwards' method, a significant cyclic trend was found in the monthly deaths from breast cancer and ovarian cancer. The significant peaks were in January and in September for breast cancer and ovarian cancer, respectively. Furthermore, significant seasonality was detected for all deaths using Edwards' method with a peak in January and a nadir in July (Figure 2).

However, applying the Walter-Elwood method which took into account the number of all deaths as the underlying population, a different pattern of significant seasonal variation was observed in all cancer groups with a peak in August and a nadir in February (Figure 3a-d.).

The logistic regression method revealed similar seasonal trends as the Walter-Elwood method for each cancer group. There was no significant double peak model of seasonality.

\section{Discussion}

\section{Main findings}

Decreasing annual trends in the ASMRs of cervical cancer and corpus uteri cancer were found during the study period. 
In contrast to Nakaji et al [10], significant seasonality was detected for overall deaths using Edward's method with a peak in January and a nadir in July. Since the largest observed numbers of deaths were in the winter months, thus it could reflect the seasonality of infectious diseases in temperate climates: respiratory virus infections (for example, influenza, parainfluenza, etc.) show marked seasonality occurring in the winter months [11].

Similarly, the majority of deaths from cancers of female genital organs occurred in winter but a significant seasonal pattern was only revealed for deaths from breast cancer with a peak in January and a nadir in July using the observed numbers of deaths.

However, seasonality in the proportion of deaths from female genital organs and breast cancers detected a different peak and nadir. Since the proportion of female genital organs and breast cancer deaths among all deaths was higher around the end of summer the Walter-Elwood and logistic regression methods showed significant seasonal variation with a peak in August and nadir in February. Thus, both seasonal patterns (deaths from cancers of female genital organs and deaths from all causes) are interesting and informative to potentiate prevention.

As far as we are aware, this is the first epidemiological study reporting the effect of seasonality for deaths from female genital organs and breast cancers.

\section{Strength and weaknesses}

The data were obtained from civil registers, which could have been influenced by a certain simplification (e.g. lack of subtype of cause of death) of categorization during the 35-year interval of the study.

The description of incidence rates in terms of seasonal variations or cyclic trends is important in many epidemiological studies. Both the Edwards and the Walter-Elwood methods are statistical tests to detect seasonality. However, the Edwards method does not use the population at risk only observed frequencies of deaths were used. Furthermore, the logistic regression 
method using both sine and cosine functions was applied to model seasonality. These functions can be incorporated in a regression model which allows the investigation of single or double peaks of seasonality. Although the underlying infectious agents are unknown, an investigation of seasonality stands as an important component in the understanding of the etiological description of certain diseases.

\section{Comparison with other studies}

Many studies have reported investigations of trends in cancer incidence and mortality in different geographical areas [12-15]. Hungarian mortality rates in breast cancer (20.7 per 100 000 persons per year), uterus cancer (3.75 per 100000 persons pear year) and ovarian cancer (7.02 per 100000 persons per year) were higher in 2012 than mortality rates in Central Europe as reported by Ferlay et al: mortality rates for breast cancer, cervical cancer, uterus cancer and ovarian cancer in 2012 were 16.5, 6.5, 3.4 and 6.0 per 100000 persons per year, respectively [15]. However, the mortality rate in cervical cancer (5.22 per 100000 persons per year) was smaller in Hungary than the Central European mortality rate [16]. Similar to Minelli et al, decreasing trends in mortality rates were found for malignant neoplasms of female cervical and uterus cancers in Hungary between 1979 and 2013 [17].

In our study, trends in mortality from breast cancer were decreasing in Hungary during the period 1998-2009 and a decreasing trend in mortality rates was found for malignant neoplasms of the uterus in Hungary between 1979 and 2013. However, the mortality rate for ovarian cancer did not decline during this period. In contrast, Kjaerbye-Thygesen et al [18] also reported a decrease of the age-standardized mortality rates of ovarian cancer from 10.8 to 9.0 per 100,000 women per year in Denmark.

Stålberg et al [19] found a 4.5-fold increased risk of mortality from ovarian cancer with exposure to infectious diseases from one to five years after diagnosis. Our findings support the 
environmental and infectious hazard in the etiology of death from cancer of the female genital organs and breast.

The Hungarian mortality rates are high for breast cancer in spite of a nationwide screening program for breast and cervical cancer which was established in Hungary in 2001 and 2003, respectively [1]. However, the effectiveness of cervical cancer screening is similar to the reports from Belgium [20].

August is a vacation period in Hungary and fewer medical staff are on duty which means that there is a heavier work load during this period. Thus, the "August-effect" could partly explain the higher mortality from genital organ cancer and breast cancer at the end of the summer [21-23]. Nevertheless, nosocomial infections could also increase the risk of the mortality among the immune deficient patients [24].

\section{Conclusion}

The main finding of our study suggests that the infectious diseases may increase the risk of the mortality among the immune deficient patents. However, further investigations are needed to refine our hypothesis on the role of infections in the etiology of cancer mortality.

To reduce the overall mortality rate in female genital organs and breast cancers and to prolong life expectancy, measures must be taken to reduce those mortality rates associated with seasonal differences. 


\section{Acknowledgements}

Margit Hartai is supported by the European Union and the State of Hungary, co-financed by the European Social Fund in the framework of TÁMOP-6.1.5-14-2015-0004 'National Excellence Program’ and TÁMOP-4.2.2.D-15/1/KONV-2015-0024.

\section{Conflict of interest}

The authors declare that they have no conflict of interest. 


\section{References}

1. Kolozsvári LR, Langmár Z, Rurik I. Nationwide screening program for breast and cervical cancers in Hungary: special challenges, outcomes, and the role of the primary care provider. Eur J Gynaecol Oncol. 2013;34:419-424.

2. Jemal A, Center MM, DeSantis C, Ward EM. Global Patterns of Cancer Incidence and Mortality Rates and Trends. Cancer Epidemiol Biomarkers Prev. 2010;19:1893-1907.

3. Altobelli E., Lattanzi A. Breast cancer in European Union: an update of screening programmes as of March 2014. Int J Oncol. 2014;45:1785-92.

4. Weiderpass E, Antoine J, Bray FI, Oh JK, Arbyn M. Trends in corpus uteri cancer mortality in member states of the European Union. Eur J Cancer. 2014;50:1675-1684.

5. Stolwijk AM, Straatman H, Zielhuis GA. Studying seasonality by using sine and cosine functions in regression analysis. J Epidemiol Community Health 1999;53:235-238.

6. The Hungarian Central Statistical Office. Demographic Yearbook, 1963-2012. Budapest, KSH. 1980-2014.

7. http://surveillance.cancer.gov/joinpoint

8. Edwards JH. The recognition and estimation of cyclic trends. Ann Hum Genet. $1961 ; 25: 83-87$.

9. Walter SD, Elwood JM. A test for seasonality of events with a variable population at risk. Br J Prev Soc Med. 1975; 29:18-21.

10. Nakaji S, Parodi S, Fontana V, Umeda T, Suzuki K, Sakamoto J, Fukuda S, Wada S, Sugawara K. Seasonal changes in mortality rates from main causes of death in Japan (1970--1999). Eur J Epidemiol. 2004;19:905-13.

11. Lopman BA, Reacher M, et al.. A summertime peak of "winter vomiting disease": surveillance of noroviruses in England and Wales, 1995 to 2002. BMC Public Health. $2003 ; 3: 13$ 
12. Althuis MD, Dozier JM, Anderson WF, Devesa SS, Brinton LA. Global trends in breast cancer incidence and mortality 1973-1997. Int J Epidemiol. 2005;34:405-12.

13. Allemani C, Weir HK, Carreira H, Harewood R, Spika D, Wang XS, et al. Global surveillance of cancer survival 1995-2009: analysis of individual data for $25,676,887$ patients from 279 population-based registries in 67 countries (CONCORD-2). Lancet. 2015;385(9972):977-1010.

14. Leclère B, Molinié F, Trétarre B, Stracci F, Daubisse-Marliac L, Colonna M; GRELL Working Group. Trends in incidence of breast cancer among women under 40 in seven European countries: a GRELL cooperative study. Cancer Epidemiol. 2013;37:544-549.

15. McPhail S, Johnson S, Greenberg D, Peake M, Rous B. Stage at diagnosis and early mortality from cancer in England. Br J Cancer. 2015;112 Suppl 1:S108-15.

16. Ferlay J, Soerjomataram I, Dikshit R, Eser S, Mathers C, Rebelo M, et al. Cancer incidence and mortality worldwide: sources, methods and major patterns in GLOBOCAN 2012. Int J Cancer. 2015;136:E359-86.

17. Minelli L, Stracci F, Prandini S, Moffa IF, La Rosa F. Gynaecological cancers in Umbria (Italy): trends of incidence, mortality and survival, 1978-1998. Eur J Obstet Gynecol Reprod Biol. 2004;115:59-65.

18. Kjaerbye-Thygesen A, Huusom LD, Frederiksen K, Kjaer SK. Trends in the incidence and mortality of ovarian cancer in Denmark 1978-2002. Comparison with other Nordic countries. Acta Obstet Gynecol Scand. 2005;84:1006-12.

19. Stålberg K, Svensson T, Lönn S, Kieler H. The influence of comorbidity on mortality in ovarian cancer patients. Gynecol Oncol. 2014;133:298-303.

20. Tjalma WA. The ideal cervical cancer screening recommendation for Belgium, an industrialized country in Europe. Eur J Gynaecol Oncol. 2014;35:211-8. 
21. Kamau C. Effects of experimental inductions for newly qualified doctors on competence at clinical procedures. Clin Med. 2014;14:380-5.

22. Vaughan L, McAlister G, Bell D. 'August is always a nightmare': results of the Royal College of Physicians of Edinburgh and Society of Acute Medicine August transition survey. Clin Med 2011;11:322-6.

23. Blakey JD, Fearn A, Shaw DE. What drives the 'August effect'? A observational study of the effect of junior doctor changeover on out of hours work. JRSM Short Rep. 2013;4:1-7.

24. Dinkel RH, Lebok U. A survey of nosocomial infections and their influence on hospital mortality rates. J Hosp Infect. 1994;28:297-304. 


\section{Figure legends}

Figure 1. The age standardized female genital organs and breast cancer mortality rates in Hungary during the period 1979-2013.

Figure 2. Significant seasonality was detected for overall deaths using Edwards' method with a peak in January and a nadir in July.

Figure 3. Seasonal variation in month of deaths from cancer of breast and female genital organs in Hungary during the period 1979-2013.

\section{Table headings}

Table 1 . Total numbers of deaths by age during the 35 years of study in Hungary.

Table 2. Age-standardized mortality rates per 100000 persons

Table 3. The monthly numbers of deaths used in seasonal analyses in Hungary during the period 1979-2013. 